\title{
Theoretical and Experimental Investigation of Direct Detection Optical OFDM Systems with Clipping and Normalization
}

\author{
Jiang $\mathrm{Wu}^{1}$ and Zhongpeng Wang $\mathbb{D}^{2,3}$ \\ ${ }^{1}$ School of Information, Zhejiang Sci-Tech University, Hangzhou 310023, China \\ ${ }^{2}$ School of Information and Electronic Engineering, Zhejiang University of Science and Technology, Hangzhou 310023, China \\ ${ }^{3}$ State Key Laboratory of Millimeter Waves, Southeast University, Nanjing 210096, China
}

Correspondence should be addressed to Zhongpeng Wang; wzp1966@sohu.com

Received 18 June 2018; Revised 22 August 2018; Accepted 5 September 2018; Published 15 October 2018

Academic Editor: Jit S. Mandeep

Copyright (c) 2018 Jiang Wu and Zhongpeng Wang. This is an open access article distributed under the Creative Commons Attribution License, which permits unrestricted use, distribution, and reproduction in any medium, provided the original work is properly cited.

\begin{abstract}
A data clipping and normalization technique is employed to improve the performance of the overall direct detection optical orthogonal frequency division multiplexing (DCO-OFDM) system. A detailed analysis of clipping distortion introduced by digital clipping and normalization is provided. The normalization operation amplifies the clipped data signal to the maximum input amplitude of a digital-to-analog converter (DAC). Based on the analysis, a BER formula of the proposed scheme is derived over the AWGN channel and single fiber channel. Performance of an optical clipped OFDM with normalization is assessed through numerical simulations and Monte Claro simulation over the AWGN channel. Theoretical analysis and simulation results both show that the clipping and normalization scheme can greatly improve the BER of an optical OFDM. In particular, BER performance of the proposed transmission scheme was measured in a practical OFDM transmission platform. The measured experimental results show that the clipped and amplified OFDM signal exhibits superior performance in comparison with the conventional OFDM signal. The received sensitivity at a BER of $10^{-3}$ for a $4 \mathrm{Gsamples} / \mathrm{s}(2.6667 \mathrm{Gbits} / \mathrm{s})$ clipped and normalized OFDM signal with clipping ratio of 4 after $100 \mathrm{~km}$ standard single-mode fiber (SMF) transmission was improved by $4.3 \mathrm{~dB}$ when compared with the conventional OFDM system. The measured results also showed that the clipped OFDM signal exhibits superior performance in comparison with the conventional OFDM signal. Therefore, a clipping and normalization at the transmitter is most effective, and a substantial performance improvement can be obtained by a simple normalization after clipping.
\end{abstract}

\section{Introduction}

In recent years, orthogonal frequency division multiplexing (OFDM) modulation has been proposed in optical communications due to its advantage of having robustness to fiber transmission impairments such as chromatic dispersion [1-3]. In general, optical OFDM communication can be categorized as coherent optical OFDM (CO-OFDM) and direct detection optical OFDM (DDO-OFDM). Due to the low cost and complexity of DDO-OFDM, DDO-OFDM is mainly considered in short-range optical communication systems [4]. However, the optical OFDM signal in both types of transmission systems has a disadvantage of high PAPR. The high peak-to-average power ratio (PAPR) can lead to larger nonlinear effects, which can cause optical signal intensity fluctuation and degrade the BER performance of systems $[5,6]$. For single-channel DDO-OFDM systems, high PAPR gives rise to fiber nonlinearity such as self-phase modulation (SPM). This results in the nonlinear phase shift induced by SPM. On the contrary, high PAPR requires large dynamic range of nonlinear devices such as digital-to-analog converters (DACs), power amplifiers, and external modulators. These add the cost of systems. Therefore, mitigating nonlinearity distortion in optical OFDM by reducing PAPR becomes the important issue. In order to reduce PAPR, many methods have been proposed for PAPR reduction, such as companding [7], clipping [8], DCT precoding [9], DFT precoding $[10,11]$, and other methods $[12,13]$. Recently, a fiber nonlinearity equalizer, which is based on support vector classification for optical OFDM, has been 
proposed [14]. Among these methods, the precoding technique draws great attention for its effective PAPR reduction. The precoding method utilizes additional DCT (or DFT) and IDCT (or IDFT) at the transmitter and receiver, respectively. So this method increases the computational complexity of the system.

Among the PAPR reduction methods, the clipping is the simplest. The idea of the clipping technique is clipping the signal components that exceed some clipping threshold. In general, the clipping technique can cause impairment to a communication system. In practical system, clipping may be employed before or after DACs. Clipping, which is employed before DACs, is called soft clipping, whereas clipping used after DACs is called hard clipping. A number of papers have studied the clipping effect on the radio frequency (RF) OFDM signals [15-17]. However, there is an important difference between DDO-OFDM and RF-OFDM: the RF baseband signal is complex-valued, whereas timedomain signal in DDO-OFDM is real-valued and nonnegative [18]. A real-valued time signal can be obtained when the Hermitian symmetry condition must be satisfied in OFDM subcarriers. After that, a DC bias is added to the realvalued signal resulting in a nonnegative valued signal for DDO-OFDM. In addition, for optical OFDM system, high PAPR can give rise to fiber nonlinearity which in turn results in performance degradation of systems. Thus, some theory and analyses in RF-OFDM are not directly applied to optical OFDM. A number of papers have analyzed the clipping effects in optical OFDM systems [8,19-21]. In [22, 23], the performance of IM/DD optical OFDM with the digital baseband distortion has been analyzed and evaluated by simulations. However, these researches concern on how to decrease the negative effect of clipping on the performance.

The study results of the literature [24] showed that the degradation in the error rates is very small for clipping with the clipping ratio of $6 \mathrm{~dB}$ in frequency selective fading channels. The researched results of the paper [25] showed that the performance of the overall communication system can be improved by a baseband clipping method. So, the benefits of the clipping on the OFDM system can be obtained by fixing the appropriate value of clipping ratio. In fact, the performance degradation of systems caused by clipping is negligible when the proper clipping lever is used, whereas obtaining PAPR reduction by clipping can help to mitigate nonlinearities effects of fiber and Mach-Zehnder modulator. However, the advantage of the nonlinearity mitigation by clipping is often be neglected in optical OFDM systems. The clipped optical OFDM signal with low PAPR may obtain two benefits compared to the conventional optical OFDM signal: one is that clipped OFDM results in nonlinear interference reduction caused by the Kerr effect in a fiber-optic channel [26-28] and the second is that clipped OFDM may reduce to the impact of the MZM nonlinear on the system [5,29]. Thus, proper clipping in optical OFDM may improve the BER of optical OFDM.

In this paper, we mainly investigate the effect of clipping DDO-OFDM systems. As we know, there is less attention on the benefits of the clipping technique for PAPR of the baseband OFDM signal in a direct detection OFDM communication system. Inspired by the idea in paper $[24,25]$, we proposed a data clipping and normalization scheme, which limits the high-power spikes signal and amplifies the clipped low amplitude signal. We drive an analytical expression for the BER of the DDO-OFDM system based on data clipping and normalization. The BER expression quantitatively shows how the data clipping and normalization scheme improves the BER performance. We compare our analytical results to simulation results in an additive white Gaussian noise (AWGN) channel. The simulation results agree well with the analytical results. In particular, BER performance of the proposed transmission scheme is measured in a practical OFDM transmission platform. The experimental results show that the BER performance of the proposed system can be greatly improved compared with the conventional optical OFDM system and conventional clipped optical OFDM system. Thus, the proposed joint data clipping and normalization technique can be used for the optical communication system design.

The paper is organized as follows. In Section 2, the principle of a direct detection optical OFDM system with data clipping and normalization is presented. In Section 3, the theoretical analysis is given and the BER formulas of the clipped and normalized optical OFDM are derived. Section 4 presents the simulation results. Section 5 provides the experimental configuration, and Section 6 gives measured results. Finally, Section 7 concludes this paper.

\section{System Principle}

In this work, we employ a joint data clipping and normalization technique in the transmitter for an optical DDOOFDM system to improve the BER performance of a system. Figure 1 illustrates the block diagram of the proposed DDOOFDM transmitter, in which $N$ subcarriers is used. At the transmitter, an incoming bit stream is first mapped into an M-ary quadrature amplitude modulation (M-QAM) symbol data stream. Then, a data symbol vector with $N$ size is formed as $X=\left[\begin{array}{llllllll}0 & X_{1} & \ldots & X_{N / 2-1} & 0 & X_{N / 2-1}^{*} & \ldots & X_{1}^{*}\end{array}\right]$. In order to produce a real-valued time-domain OFDM signal $x_{n}$, the input of the IFFT $X_{k}$ is Hermitian symmetric, that is,

$$
\begin{aligned}
& X_{k}=X_{N-k}^{*}, \quad 1 \leq k \leq N-1, \\
& X_{k}=0, k=0, \quad N / 2,
\end{aligned}
$$

where $*$ denotes complex conjugate. Thus, performing an $N$-point IFFT on $X$ produces real-valued signal $x$, that is,

$$
x_{n}=\frac{1}{\sqrt{N}} \sum_{k=0}^{N-1} X_{k} \exp \left(\frac{j 2 \pi k n}{N}\right), \quad 0 \leq n \leq N-1 .
$$

After IFFT, the OFDM signal is fed into the data clipping blocks, where the signal is clipped by the data clipping block. We consider that the symmetric clipping is used. The operation of the data clipping (or baseband clipping) is represented as follows [31]: 


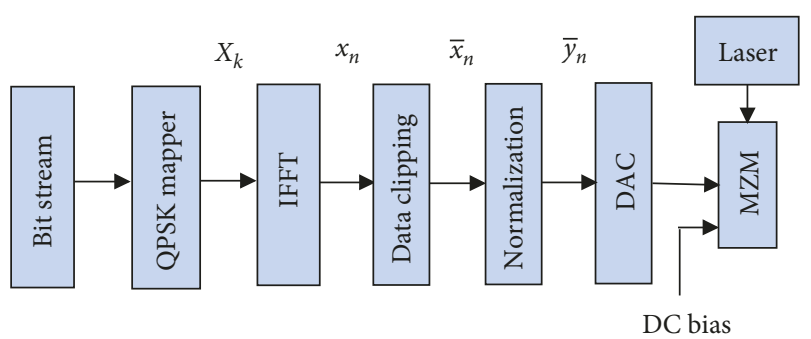

FIGURE 1: Block diagram of the proposed OFDM transmitter.

$$
\bar{x}=g\left(x_{n}\right)= \begin{cases}x_{n} & \text { if }\left|x_{n}\right| \leq A_{\text {clip }}, \\ A_{\text {clip }} \frac{x_{n}}{\left|x_{n}\right|} & \text { if }\left|x_{n}\right| \geq A_{\text {clip }} .\end{cases}
$$

The clipping ratio (CR) is defined by the following equation:

$$
\mathrm{CR}=\frac{A_{\text {clip }}^{2}}{P_{x}}
$$

where $P_{x}$ is the average power of the transmitted signal and $A_{\text {clip }}$ is the maximum allowed signal amplitude of the clipped OFDM signal, and it can be written as

$$
A_{\text {clip }}=\sqrt{C R \cdot P_{x}} \text {. }
$$

Higher clipping level values can be applied to the signal for reducing the clipping noise at the expense of increasing the electrical power of the signal. Hence, there is a trade-off between power efficiency and noise in the selection of the clipping level or bias. When $A_{\text {clip }}$ is smaller than the maximum allowable amplitude of a DAC device, $A_{\max }$, the system performance can be improved by normalizing the clipped OFDM signal. The clipped and normalized signal, which is the output of the normalization, is written as

$$
\bar{y}_{n}=\frac{A_{\max }}{A_{\text {clip }}} \bar{x}= \begin{cases}G x_{n} & \text { if }\left|x_{n}\right| \leq A_{\text {clip }}, \\ G\left(A_{\text {clip }} \frac{x_{n}}{\left|x_{n}\right|}\right) & \text { if }\left|x_{n}\right| \geq A_{\text {clip }}\end{cases}
$$

where $1 \leq G \leq\left(A_{\max } / A_{\text {clip }}\right) . G$ is the normalized factor. The maximum allowable normalized factor can be expressed as

$$
G_{\max }=\frac{A_{\text {max }}}{A_{\text {clip }}}=\frac{A_{\max }}{\sqrt{\mathrm{CR} \cdot P_{x}}}=\frac{A_{\max }}{\sqrt{P_{x}}} \cdot \mathrm{CR}^{-1 / 2} .
$$

Obviously, the smaller the value of clipping ratio is, the higher the normalization factor is. From Equation (6), we can see that the small signal, whose amplitude is below threshold A, is amplified by $G$ times. When $G=1$, the output of the normalization is the conventional clipped OFDM signal. In order to utilize fully the dynamic range of a DAC device, the clipped signal $\bar{x}(n)$ is normalized to $\bar{y}(n)$ with a peak-to-peak of $2 A_{\max }$, which is the maximum input amplitude of a DAC device. The signal $\bar{y}(n)$ is converted into the continuous OFDM signal $y(t)$ by a DAC device in order to drive a Mach-Zehnder modulator (MZM).
According to Bussgang's theorem [30], any nonlinear function of $x(n)$ can be decomposed into a scaled version of $x(n)$ plus a distortion term $d(n)$ that is uncorrelated with $x(n)$. For example, the clipped signal can be written as

$$
\bar{x}(n)=\alpha x(n)+d(n)
$$

Scaling factor $\alpha$ can be calculated as follows [30]:

$$
\alpha=1-e^{-\mathrm{CR}}+\frac{\sqrt{\pi}}{2} \sqrt{\mathrm{CR}} \cdot \operatorname{erfc}(\sqrt{\mathrm{CR}}) .
$$

Then, the clipped signal $\bar{x}(n)$ is amplified by $G$ times, and it can be written as

$$
\bar{y}(n)=G \cdot \bar{x}(n)=G \cdot \alpha \cdot x(n)+G \cdot d(n) .
$$

In the optical link, the electrooptic conversion is performed by a standard Mach-Zehnder modulator (MZM) biased at the quadrature point. The signal $y(t)$ is biased with a biased voltage $V_{\mathrm{DC}}$ in order to ensure the waveform is nonnegative, and then, the signal is intensity modulated onto the optical carrier. The biased and clipped time-domain signal can be expressed as $\tilde{y}(t)=y(t)+V_{\mathrm{DC}}$. This signal $\bar{y}(t)$ is then used to drive an idea optical modulator, where the output optical power is a replica of the corresponding electrical-drive signal.

The fiber nonlinearity remains sufficiently low when the optical launch power is not too high. In this case, the optical fiber link can be modeled as a linear channel [31]. Hence, the optical signal and noise can be assumed independent. Similar to the analysis in [31], polarization-mode dispersion (PMD) and fiber nonlinearity are neglected, and groupvelocity dispersion (GVD) is the only fiber impairment considered. In the optical electric field domain, the fiber is modeled as a linear system with the transfer function given by Barros and Kahn [31]:

$$
H(\omega)=e^{j \omega^{2}\left(\beta_{2} / 2\right) L},
$$

where $\beta_{2}$ is the fiber GVD parameter and can be expressed as $\beta_{2}=-D \lambda^{2} / 2 \pi c$. Here, $\lambda$ represents the carrier wavelength, and $c$ is the speed of light. In addition, $L$ is the fiber length, and $\omega$ is the angle frequency. Assume $h(t)$ is the impulse response of the fiber channel, and it equal to the inverse Fourier transformation of $H(\omega)$.

At the receiver, the $\mathrm{PD}$ detector converts the received optical signal to electrical signal. The sampled discrete signal can be expressed as

$$
\begin{aligned}
r(n) & =\bar{y}(n) \otimes h(n)+w(n) \\
& =G \bar{x} \otimes h(n)+w(n) \\
& =G a x(n) \otimes h(n)+G d(n) \otimes h(n)+w(n),
\end{aligned}
$$

where $w(n)$ is AWGN and $\otimes$ denotes circular convolution. By taking the DFT of Equation (12), the frequency domain data on the $k$ th subarrier can be written as

$$
R_{k}=G \alpha X_{k} H_{k}+G D_{k} H_{k}+W_{k},
$$

where $H_{k}$ is the channel frequency response on the $k$ th subcarrier and $W_{k}$ is the additive white Gaussian noise in the 
frequency domain. When zeros forcing (ZF) equalization is employed, the coefficient of the $k$ th subcarrier is given by

$$
\mathrm{Q}_{\mathrm{ZF}, k}=\frac{1}{H_{k}}, \quad k=0,1, \ldots, N-1 .
$$
as

After equalization, the received signal can be expressed

$$
\widehat{R}_{k}=G \alpha X_{k}+G D_{k}+H_{k}^{-1} W_{k}
$$

\section{Theoretical Analysis}

3.1. BER Performance over AWGN Channel. In this subsection, we firstly analyze the BER performance of the proposed transmission scheme for the AWGN channel. At the receiver of the proposed system, the clipped OFDM signal with normalization after transmission over the AWGN channel can be expressed as

$$
\begin{aligned}
r(n) & =G \cdot \bar{x}(n)+w(n), \\
& =G \cdot \alpha \cdot x(n)+G \cdot d(n)+w(n) .
\end{aligned}
$$

The frequency signal at the $k$ th subcarrier can be given as

$$
R_{k}=G \alpha X_{k}+G D_{k}+W_{k} \text {. }
$$

Obviously, the clipping operation reduces the power of the signal. The loss power due to clipping can be estimated. It is can be given by the following equation [32]:

$$
\sigma_{d}^{2}=2 \cdot \sqrt{\frac{2}{\pi}} \cdot \sigma_{x}^{2} \mathrm{CR}^{-3 / 2} e^{-\mathrm{CR} / 2}
$$

Base on Equation (16), the SNR of the OFDM signals can be expressed as

$$
\lambda=\frac{\alpha^{2} G^{2} \sigma_{x}^{2}}{G^{2} \sigma_{c}^{2}+\sigma_{0}^{2}}=\frac{\alpha^{2} \sigma_{x}^{2}}{\sigma_{d}^{2}+\left(\sigma_{0}^{2} / G^{2}\right)} .
$$

In order to take the useful dynamic range of a DAC, the allowable maximum normalization gain $G_{\max }$ in Equation (7) is adopted. By substituting Equations (7) and (18) into Equation (19), the SNR under the maximum normalization gain can be written as

$$
\begin{aligned}
\lambda^{a m} & =\frac{\alpha^{2} \sigma_{x}^{2}}{\sigma_{d}^{2}+\sigma_{0}^{2} / G^{2}}, \\
& =\frac{\left[1-e^{-\mathrm{CR}^{2}}+(\sqrt{\pi} / 2) \sqrt{\mathrm{CR}} \cdot \operatorname{erfc}(\sqrt{\mathrm{CR}})\right]^{2} \cdot \sigma_{x}^{2}}{2 \cdot \sqrt{2 / \pi} \cdot \sigma_{x}^{2} \mathrm{CR}^{-3 / 2} e^{-\mathrm{CR} / 2}+\sigma_{0}^{2} \cdot \sigma_{x}^{2} \cdot\left(\sqrt{\mathrm{CR}} / A_{\max }^{2}\right)} .
\end{aligned}
$$

Thus, the theoretical BER formula of the clipped and normalized M-QAM OFDM can be expressed from [33]:

$$
P_{b, \mathrm{MQAM}}^{a m}=\left(\frac{4-2^{(2-m / 2)}}{m}\right) Q\left(\sqrt{\frac{3}{(M-1)} \lambda^{a m}}\right),
$$

where $m=\log _{2} M$ is the number of bits per constellation point. The $Q$ function is defined as

$$
Q(x)=\frac{1}{\sqrt{2 \pi}} \int_{x}^{\infty} e^{-t^{2} / 2} d t .
$$

When $G=1$, Equation (20) becomes the SNR of the conventional clipped OFDM, and it can be expressed as

$$
\lambda^{c}=\frac{\left[1-e^{-\gamma^{2}}+\sqrt{\pi} / 2 \gamma \cdot \operatorname{erfc}(\gamma)\right]^{2} \cdot \sigma_{x}^{2}}{2 \cdot \sqrt{2 / \pi} \cdot \sigma_{x}^{2} \gamma^{-3} e^{-\gamma^{2} / 2}+\sigma_{0}^{2}} .
$$

Therefore, the theoretical BER expression of conventional OFDM over the AWGN channel is given from [33]:

$$
P_{b, \mathrm{MAQM}}=\left(\frac{4-2^{(2-m / 2)}}{m}\right) Q\left(\sqrt{\frac{3}{(M-1)} \lambda^{c}}\right) \text {. }
$$

3.2. BER Performance for Single-Mode Fiber Channel. Based on Equation (15), the signal-to-noise-and-distortion ratio (SNDR) of the $k$ th subcarrier for the clipped and normalized OFDM system is given by

$$
\begin{aligned}
\lambda_{k, G}^{\mathrm{ZF}} & =\frac{E\left[\left|G \alpha X_{k}\right|^{2}\right]}{E\left[\left|G D_{k}+H_{k}^{-1} W_{k}\right|^{2}\right]}=\frac{G^{2} \alpha^{2} \cdot \sigma_{X}^{2}}{G^{2} \sigma_{D}^{2}+H_{k}^{-2} \sigma_{W}^{2}} \\
& =\frac{\alpha^{2} \cdot \sigma_{X}^{2}}{\sigma_{D}^{2}+\left(1 / G^{2}\right) \cdot\left(\sigma_{W}^{2} / H_{k}^{2}\right)},
\end{aligned}
$$

where $\sigma_{X}^{2}$ is the variance of the signal $X_{k}, \sigma_{D}^{2}$ is the variance of the signal $D_{k}$, and $\sigma_{W}^{2}$ is the variance of the AWGN noise $W_{k}$.

Thus, the BER performance of every subcarrier can be expressed as

$$
P_{b, k}^{a m}=\left(\frac{4-2^{(2-m / 2)}}{m}\right) Q\left(\sqrt{\frac{3}{(M-1)} \lambda_{k, G}^{\mathrm{ZF}}}\right) .
$$

The overall performance of the clipped and normalized system can be expressed as

$$
P_{b}^{a m}=\frac{1}{N} \sum_{n=0}^{N-1} P_{b, k}^{a m} .
$$

When $G=1$ in Equation (25), the clipped and normalized OFDM system is converted into the conventional clipped OFDM system. Then, the signal-to-noise-anddistortion ratio (SNR) of the $k$ th subcarrier at the receiver for the conventional clipped OFDM system is given by

$$
\lambda_{k}^{\mathrm{ZF}}=\frac{E\left[\left|\alpha X_{k}\right|^{2}\right]}{E\left[\left|D_{k}+H_{k}^{-1} W_{k}\right|^{2}\right]}=\frac{\alpha^{2} \cdot \sigma_{X}^{2}}{\sigma_{D}^{2}+\left(\sigma_{W}^{2} / H_{k}^{2}\right)} .
$$

Therefore, the BER of every subcarrier of the clipped system without normalization can be expressed as

$$
P_{b, k}=\left(\frac{4-2^{(2-m / 2)}}{m}\right) Q\left(\sqrt{\frac{3}{(M-1)} \lambda_{k}^{Z F}}\right) .
$$

The overall performance of the clipped OFDM system can be expressed as 


$$
P_{b}=\frac{1}{N} \sum_{n=0}^{N-1} P_{b, k}
$$

Compared with Equations (25) and (28), when G>1, we can conclude that

$$
\lambda_{k, G}^{\mathrm{ZF}} \geq \lambda_{k}^{\mathrm{ZF}} .
$$

Furthermore, there is $P_{b}^{a m}<P_{b}$. Thus, a joint clipping and normalization scheme can be employed in optical OFDM in order to improve BER performance. The BER of the simulation and measured results in the following section show that the BER performance of the clipped and normalized OFDM signal can be greatly improved compared with the conventional clipped OFDM and conventional OFDM signals.

\section{Simulation Results}

In the following simulation, there are two criterions: equal average power and equal maximum amplitude criterions. The equal average power criterion is employed in many PAPR reduction techniques. In this case, the clipping obtains PAPR reduction at cost of degradation in BER performance. Obviously, the clipping decreases the average power of the OFDM signal, and the BER performance can be slightly improved if the loss average power of the signal can be compensated. In most reported literatures, the negative effect of clipping on BER performance has been widely concerned. Many techniques such as channel coding and iterated signal detector are employed to reduce the effect of clipping on systems. However, the effect of clipping on BER is not severe when the clipping ratio is greater than $6 \mathrm{~dB}$.

4.1. Q Factor of the Clipped OFDM by Simulation. Q factor usually serves as another metric to evaluate the quality of signals in terms of its SNR. The higher the value of $Q$ factor, the lower the bit error rate (BER). We first research the effect of the clipping with and without normalization on the $Q$ factor of signals. The measured or estimated BER is also expressed as a $Q$ factor using the following equation [34]:

$$
Q(\mathrm{~dB})=20 \log \left(\sqrt{2} \times \operatorname{erfc}^{-1}(2 \times \mathrm{BER})\right),
$$

where $\mathrm{erfc}^{-1}$ is the inverse complementary error function. Based on Equation (32), the obtained BER can be converted to the corresponding $Q$ factor in $\mathrm{dB}$.

Figure 2 shows the relationship between $Q$ factor and clipping ratio (CR) over the AWGN channel at the received SNR of $6 \mathrm{~dB}$. The clipping ratio is varied from 2 to 12 , and thus, the $Q$ factor simulation results are taken. From the graph, it is analyzed that, for the clipping and normalization scheme, as the clipping ratio increases, the $Q$ factor increases when the value of clipping ratio is smaller than 4 , but it decreases when the value of clipping ratio is higher than 4 . The maximum $Q$ factor was found to be $12 \mathrm{~dB}$ at the optimum clipping ratio of 4 . However, for the conventional clipping scheme, the $Q$ factor increases, when the value of clipping ratio is below 6, but the $Q$ factor was found to be

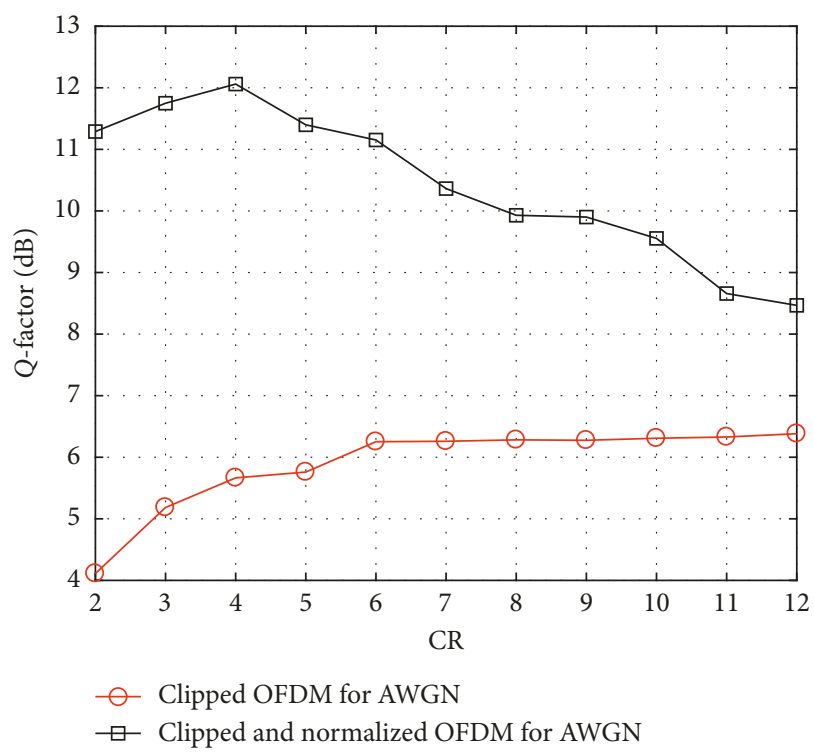

Figure 2: $Q$ factor values as a function of the clipping ratio in clipped QPSK OFDM with and without normalization over the AWGN channel.

about $6.2 \mathrm{~dB}$ at clipping ratio of 6 , but it does not almost increase with the increasing of clipping ratio. Therefore, the clipped and normalized OFDM system can obtain the best BER performance over the AWGN channel at clipping ratio (CR) of 4.

For a given received SNR of $8 \mathrm{~dB}$, Figure 3 shows the relationship of the $Q$ factor between clipping ratio in clipped QPSK OFDM with and without normalization after $100 \mathrm{~km}$ fiber transmission. The results which are similar to that of Figure 2 can also be obtained. The maximum $Q$ factor value of the clipped OFDM signal with normalization was found to be $12 \mathrm{~dB}$ at clipping ratio of 4 . The $Q$ factor decreases with increase of the clipping ratio when the value of clipping ratio is higher than 4. For the conventional clipped OFDM, the $Q$ factor increases when the value of clipping ratio varies from 2 to 6 . However, the improvement $Q$ factor is very little when the value of the clipping ratio varies from 6 to 12. Therefore, similar to Figure 2, the OFDM system can obtain the best BER performance over $100 \mathrm{~km}$ fiber channel at clipping ratio (CR) of 4.

From the experiment results in Figures 2 and 3, it can be seen that data normalization can improve the quality of the clipped OFDM signals over AWGN and fiber channel. The benefit of clipping can be achieved by data normalization. Therefore, clipping based on normalization can offer better BER as compared to the conventional clipping with the same level clipping ratio. This is also be verified by following experiments.

4.2. BER Performance over $A W G N C h a n n e l$. In the following subsection, we first evaluated the BER performance over the AWGN channel by theoretical numerical and simulation methods under without normalization case. Figure 4 shows the BER comparison for different clipping ratios for clipped OFDM without the normalization case. In this case, the 


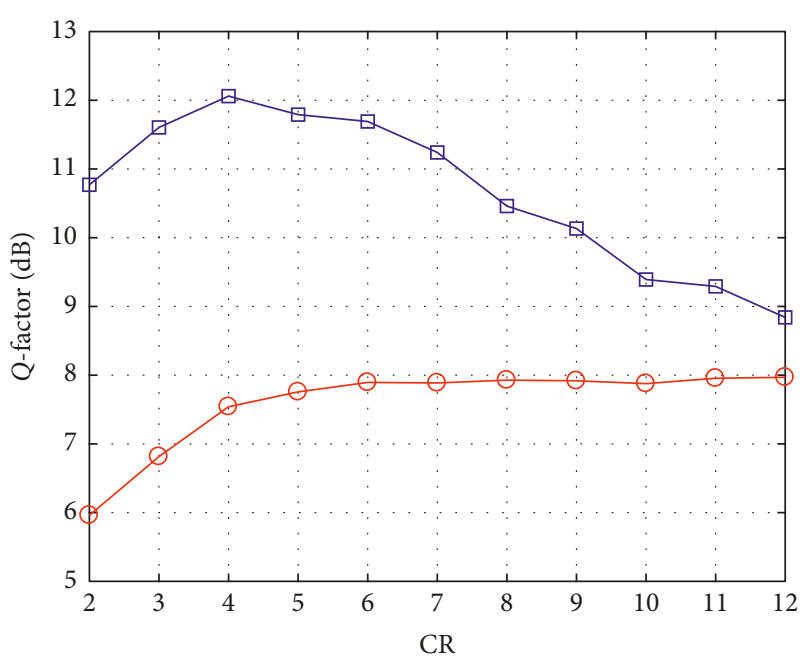

- Clipped OFDM for fiber channel

$\square$ Clipped and normalized OFDM for fiber channel

FIgURE 3: $Q$ factor values as a function of the clipping ratio in clipped QPSK OFDM with and without normalization over the fiber channel, and the transmission is $100 \mathrm{~km}$.

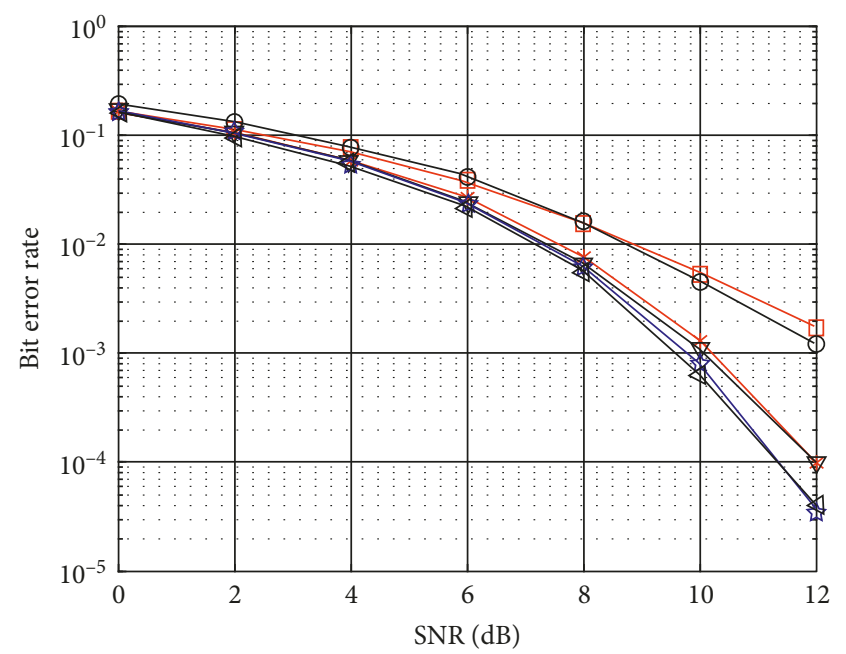

$\square$ Clipped OFDM with CR $=4$ (theoretical)

- Clipped OFDM with $\mathrm{CR}=4$ (simulation)

* Clipped OFDM with CR $=6$ (theoretical)

$\nabla$ Clipped OFDM with CR $=6$ (simulation)

Clipped OFDM with $\mathrm{CR}=10$ (theoretical)

$\dashv$ Clipped OFDM with CR $=10$ (simulation)

FIGURE 4: BER performance comparison for different clipping ratios by simulation and theoretical methods over the AWGN channel without the normalization case.

clipping degraded the BER of systems. The theoretical numerical results also are shown in Figure 4 . It is clear that simulation results agree well the theoretical results. The experiment results verify the validity of Equation (24). The performances of the clipped OFDM with normalization are compared in Figure 5 for the AWGN channel by theoretical numerical and simulation. The experiment results show that the joint data clipping and normalization can improve the

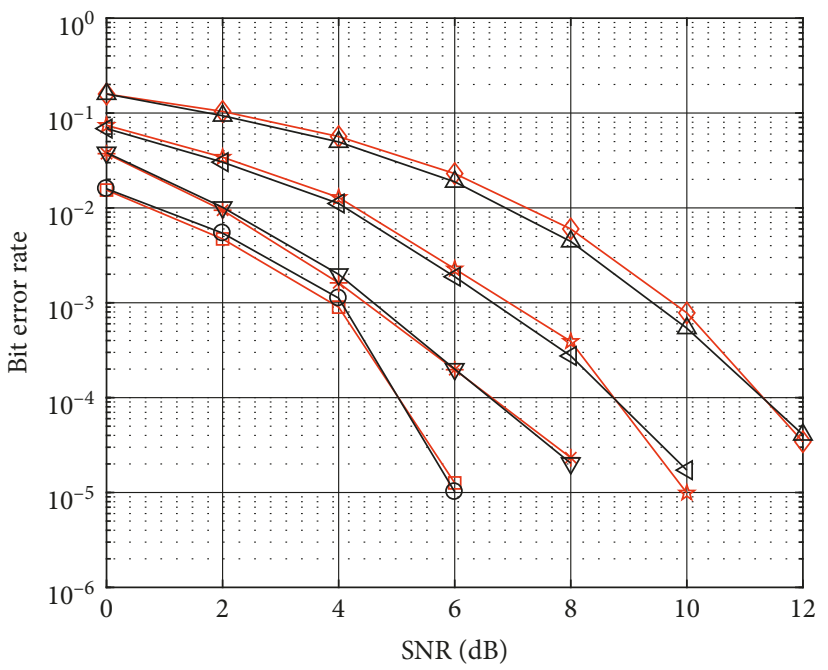

$\square$ Clipped and normalized OFDM with CR $=4$ (theoretical)
- Clipped and normalized OFDM with CR $=4$ (simulation)
$*$ Clipped and normalized OFDM with CR $=6$ (theoretical)
$\nabla$ Clipped and normalized OFDM with CR $=6$ (simulation)
位 Clipped and normalized OFDM with CR $=10$ (theoretical)
$\varangle$ Clipped and normalized OFDM with CR $=10$ (simulation)
$\diamond$ Original OFDM (theoretical)
$\triangle$ Original OFDM (simulation)

FIGURE 5: BER performance comparison for different clipped and normalized OFDM by simulation and theoretical methods over the AWGN channel.

BER performance of systems. The simulation results agree with the theoretical numerical results. These also verify the effectiveness of Equation (21).

4.3. BER Performance over Fiber Channel. For a short distance fiber-optic system with low data rate, fiber dispersion is the main impairment of degrading system performance. The optical signals affected by fiber nonlinearity are minimal. Therefore, in our simulation experiment, we neglect the effect of fiber nonlinearity so that we focus on studying how to improve the system performance by combating dispersion impairment. We evaluated the BER performance of clipped OFDM over the fiber channel with and without normalization. The main simulation parameters are shown in Table 1. Some of the parameters of the OFDM system are given according to the 802.16a OFDM physical layer (PHY) specification [35]. The number of points of FFT/IFFT, number of data subcarriers, and pilot subcarriers are fixed at 256,192 , and 8 . In addition, 56 null subcarriers serve as the guard band in order to enable the signal to naturally decay and create the FFT brick wall shaping [35]. Cyclic prefixing (CP) with 32 samples symbols is inserted to combat intersymbol interference (ISI) due to channel dispersion. We use $D$ and $\lambda$ to state the dispersion coefficient and wavelength of continuous lightwave (CW) laser, respectively.

Figure 6 shows the simulation results for clipped optical OFDM without normalization over the fiber channel. From Figure 6, we can see that the clipping can degrade the BER 
TABLE 1: Simulation parameters.

Dispersion coefficient, $D$

Optical carrier wavelength $(\lambda)$

Modulation

Sample rate

FFT size

Number of data subcarriers

Number of pilot subcarriers

Null subcarriers

Length of CP

$L$ (length of the fiber)

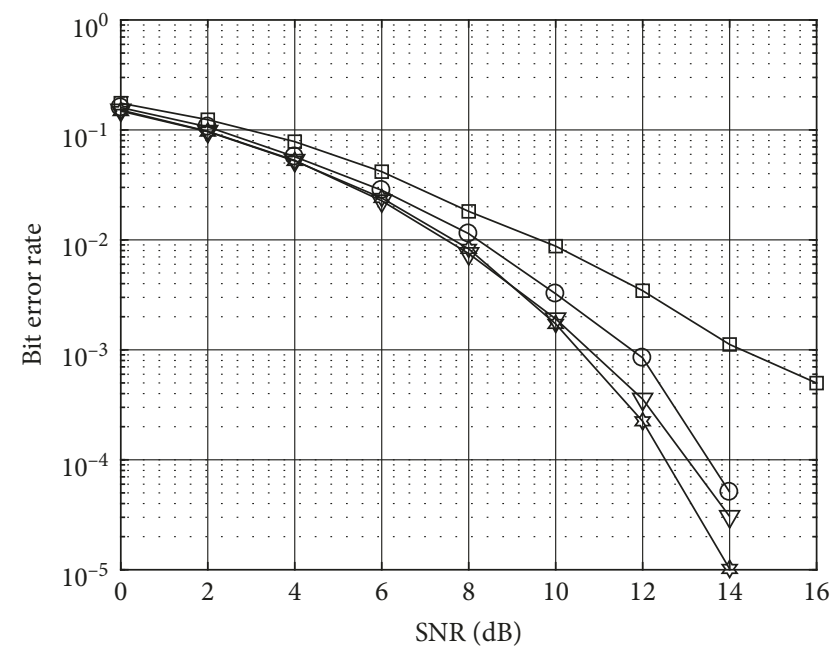

$\square$ Clipped OFDM with CR $=4$ (simulation)

- Clipped OFDM with CR $=6$ (simulation)

$\nabla$ Clipped OFDM with CR $=10$ (simulation)

Original OFDM (simulation)

FIGURE 6: BER performance comparison for different clipped and normalized OFDM without normalization over the fiber channel.

performance under without normalization. But the effect of clipping on BER performance is very small when clipping ratio is bigger than $10 \mathrm{~dB}$. At the bit error rate (BER) $10^{-3}$, the BER performance can be degraded about 3.5, 1.3, and $0.2 \mathrm{~dB}$ for clipped OFDM with clipping ratio of 4,6 , and 10 compared to that of the original OFDM.

Figure 7 shows simulation results of the clipped and normalized OFDM over the fiber channel. It can be seen that the normalization can greatly improve the BER performance of systems. At BER $10^{-3}$, the BER performance of the clipped and normalized OFDM can improve about 5.2, 5.1, and $2.4 \mathrm{~dB}$ SNR gain than original OFDM for CR of 4,6 , and 10 , respectively.

However, the obtained performance improvement is at the cost of average power increasing. All different clipped and amplified OFDM signals are amplified to the same maximum amplitude, which is the maximum allowable amplitude of DAC in the transmitter end. The more the normalization factor is, the lower the clipping ratio is. Meantime, the higher the average power is, the lower the clipping ratio is.

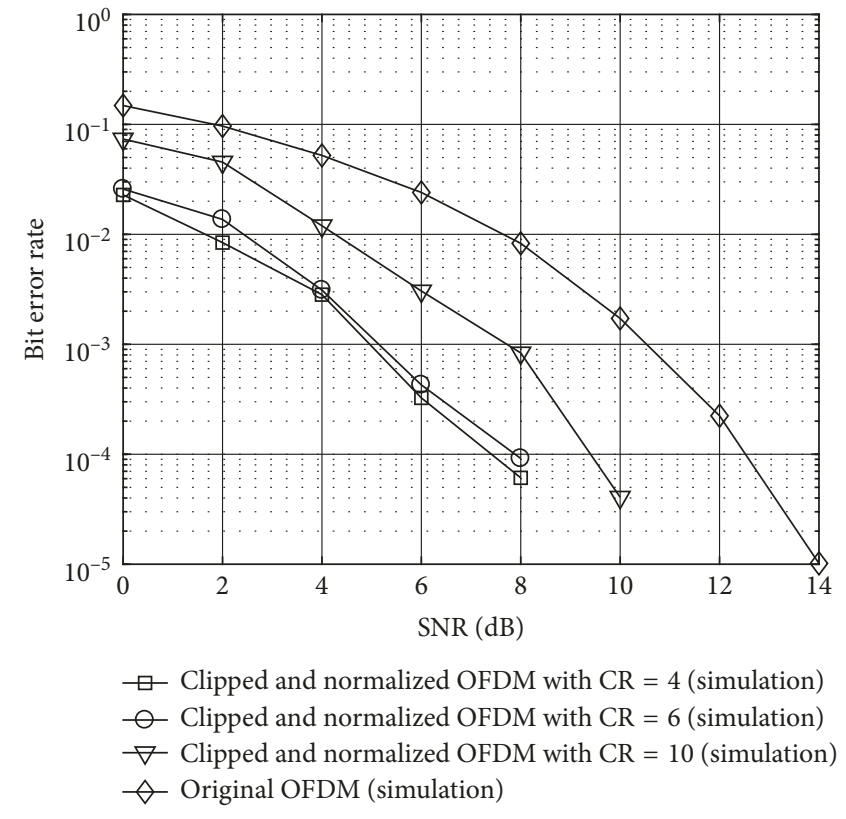

FIGURE 7: BER performance comparison for different clipped and normalized OFDM over the fiber channel by simulation.

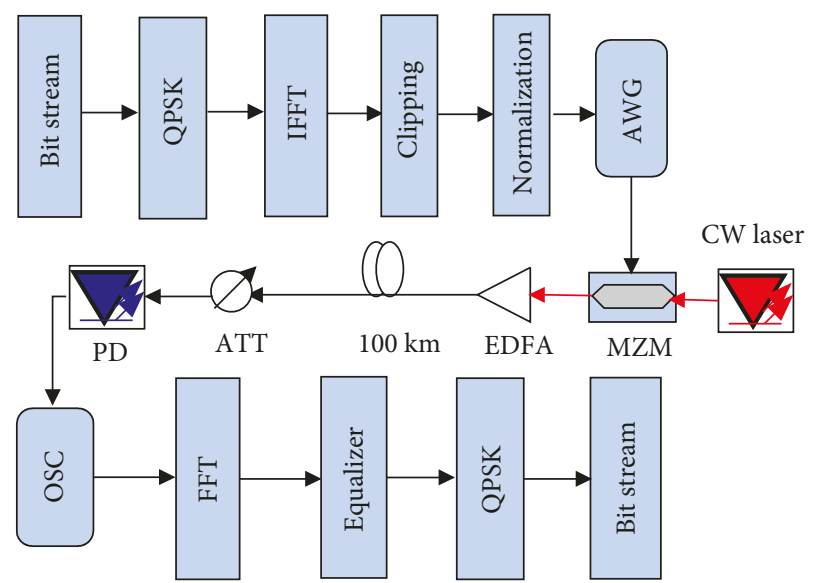

Figure 8: Experimental setup (EDFA: erbium-doped fiber amplifier; ATT: attenuator; PD: photodiode; OSC: oscilloscope; AWG: arbitrary waveform generator; ATT: attenuator).

\section{Experimental Setup}

Figure 8 depicts the experimental setup employed to evaluate the performance improvement of an optical direct detection OFDM system provided by the data clipping and normalization technique. In our experiment, $4 \mathrm{Gs} / \mathrm{s}$ OFDM signals are employed. An OFDM frame based on QPSK modulation has 256 subcarriers, among which $192(96 * 2)$ subcarriers are used for data transmission, 8 pilot subcarriers are used for channel estimation and synchronization, and 56 subcarriers are set to zero as the guard interval. In addition, 32 samples symbols are used as cyclic prefixing to avoid the interblock interference.

The time-domain QPSK OFDM waveforms are first generated in a Matlab program and uploaded onto an 
arbitrary waveform generator (AWG) operated at $4 \mathrm{GS} / \mathrm{s}$ to generate the corresponding OFDM analog signal with the peak-peak value of $1 \mathrm{~V}$. The net bit rate was $4 \mathrm{Gs} / \mathrm{s} * 192 / 2 / 256 * 256 /(256+32) * 2$ (bits/symbol for QPSK) $=2.6667 \mathrm{Gbits} / \mathrm{s}$. Then, the analog OFDM signal is modulated via an external Mach-Zehnder modulator (MZM). The MZM is biased at the quadrate point with a biased voltage of $2.2 \mathrm{~V}$. In the experiment, the value of $V_{\pi}$ is setup at $3.5 \mathrm{~V}$. The optical signal is obtained by using a continuous lightwave $(\mathrm{CW})$ laser with nominal wavelength of $1549.261 \mathrm{~nm}$. The generate signal is injected into erbiumdoped fiber amplifier (EDFA) with a noise figure of $5 \mathrm{~dB}$ to adjust the proper launched power into fiber. Then, the resulting optical signal is launched into a $100 \mathrm{~km}$ standard single-mode fiber (SSMF). The attenuation and dispersion coefficients of the fiber are $0.19 \mathrm{~dB} / \mathrm{km}$ and $17 \mathrm{ps} /(\mathrm{nm} \mathrm{km})$, respectively. At the receiver side, the signal is sampled using a digital storage oscilloscope (DSO) with $10 \mathrm{Gsamples/s}$ and applied to the OFDM demodulator. After demodulation, the received signal is equalized and applied to demapper.

\section{Experimental Results}

In this section, we will measure the performance of the clipped QPSK OFDM signal of $4 \mathrm{Gs} / \mathrm{s}$ with and without normalization after $100 \mathrm{~km}$ standard SMF transmission in our transmission experiment platform. The measured experiment results will verify that a joint clipping and normalization scheme can improve the BER performance of the system.

\subsection{Q Factor of the Clipped OFDM with Normalization.} Figure 9 shows $Q$ factor versus launch optical power after $100 \mathrm{~km}$ transmission for various values of CR. For the clipped and normalized OFDM system, the optimum $Q$ factor is about $12.7 \mathrm{~dB}$ with launch power $6 \mathrm{dBm}$ for clipped OFDM with CR of 4 . However, the optimum $Q$ factor is $10.2 \mathrm{~dB}$ with launch power $6.0 \mathrm{dBm}$ for the $4 \mathrm{Gs} / \mathrm{s}$ original OFDM signal. At low fiber launch power, the transmission performance is mainly by amplified spontaneous (ASE) noise. Hence, the $Q$ factor is improved with an increase in the fiber launch power, as shown in Figure 9. On the contrary, when the fiber launch power is over $6 \mathrm{dBm}$ for the proposed system, the $Q$ factor is decreased with an increase in the fiber launch power due to the impact of fiber nonlinearity.

High launch optical power can cause the fiber nonlinearity impairments, which includes self-phase modulation (XPM), cross-phase modulation (XPM), and four shift mixing (FWM). The nonlinear distortion can be approximated as a phase shift of the transmission signal. And the resulting phase shift causes the degradation of the performance of an optical system. The introduced shift phase shift can be expressed as follows [36]:

$$
\phi_{\mathrm{NL}}(t)=\gamma L_{\mathrm{eff}} P(t),
$$

where $\gamma$ is the nonlinear coefficient of the fiber, $P(t)$ is the instantaneous optical power, and $L_{\text {eff }}$ is the nonlinear effective length and is expressed as follows [36]:

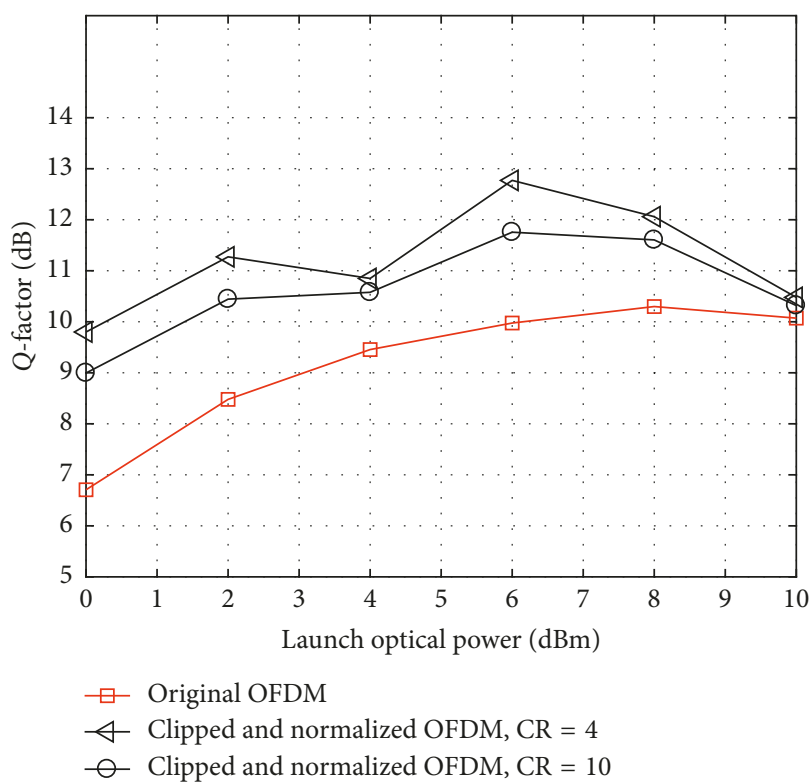

FIgURE 9: $Q$ factor values as a function of the launch power in $4 \mathrm{Gs} / \mathrm{s}$ QPSK DCO-OFDM after $100 \mathrm{~km}$ of transmission.

$$
L_{\text {eff }}=\frac{1-e^{-\alpha L}}{\alpha},
$$

where $L$ is the length of fiber and $\alpha$ is the attenuation coefficient.

Based on Equation (33), the greater the launch power, the greater the phase shift, and therefore, the lower the $Q$ factor. When the fiber nonlinearity is not the main impairment of degrading system performance, the effect of fiber nonlinearity can be neglected. From Figure 9, it can be seen that the clipped and amplified OFDM with clipping ratio of 4 can obtain the $Q$ factor improvement of $2.9 \mathrm{~dB}$ when compared to that of the original OFDM under launch optical power of $6 \mathrm{~dB}$ case. When the launch optical power is higher than the optimum value of $6 \mathrm{~dB}$, the $Q$ factor value begins to decline due to the fiber nonlinearity.

From Figure 9, the measured experiment results confirm that the joint data clipping and normalization scheme can greatly improve the BER performance of systems. The approach only is done in the digital baseband and does not need any hardware equipment.

6.2. BER of the Clipped OFDM without Normalization. Figure 10 shows the measured BER performance results at the launched optical power of the $6 \mathrm{dBm}$ case for the conventional clipped OFDM systems. As shown in Figure 3, the clipped system has excellent transmission performance because of the fiber nonlinearity mitigation by clipping when compared with the conventional OFDM system. Compared with the conventional OFDM system at $\mathrm{BER}=10^{-3}$, the data clipping can improve the BER performance approximately $1.3,2.2,0.8$, and $0.5 \mathrm{~dB}$ with $\mathrm{CR}$ at $4,6,8$, and 10 , respectively. From Figure 10, it can be seen that the optimum power clipping ratio is 6 under without normalization. In the optimum clipping case, the performance of the system can 


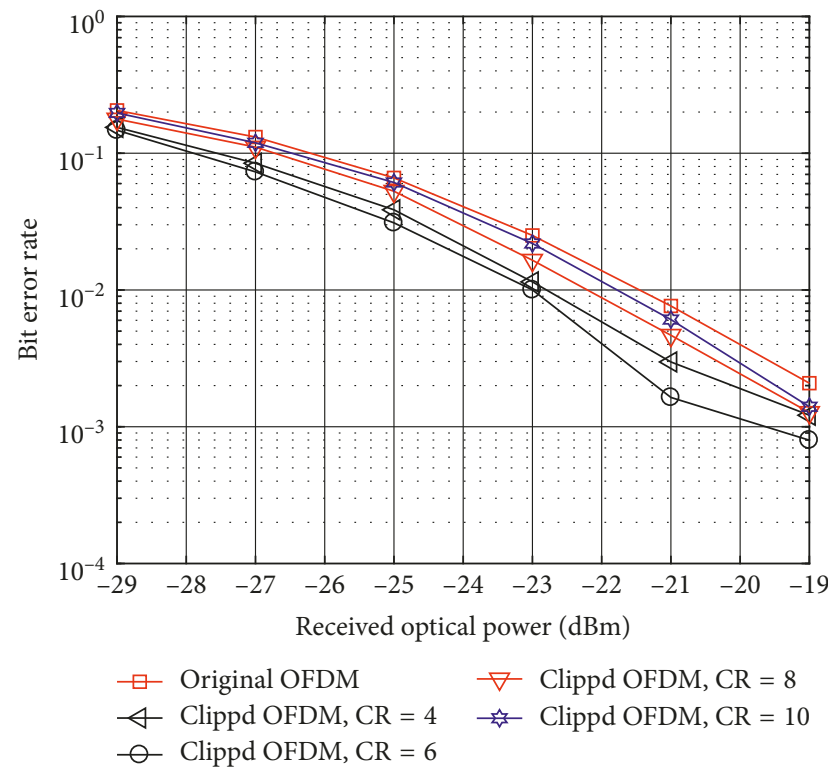

FIGURE 10: Comparison of the BER performance of the clipped QPSK OFDM signals with the launch power of $6 \mathrm{dBm}$.

obtain the greatest improvement. This is because that the decreasing of PAPR of clipped OFDM is helpful to reduce influence of nonlinearities of the fiber and MZM modulator on the BER performance of the optical OFDM systems $[26,27]$. The results of Figure 10 show that proper clipping can improve the BER performance of optical OFDM transmission systems.

\subsection{BER of the Clipped OFDM with Normalization.} Figure 11 shows the measured BER performance comparisons of the clipped and normalized OFDM signals with the launch power of $6 \mathrm{dBm}$ at $\mathrm{CR}$ of $4,6,8$, and 10 and the normalization gain is $G_{\max }=\left(A_{\max } / \sqrt{P_{x}}\right) \cdot \mathrm{CR}^{-1 / 2}$. In fact, the lower the value of $\mathrm{CR}$, the bigger the gain $G$ is.

From Figure 11, we can see that the sensitivity of the received signals of the clipped and normalized OFDM system can be improved about $4.3,4,3.5$, and $3 \mathrm{~dB}$ with $\mathrm{CR}$ of $4,6,8$, and 10 compared with conventional OFDM, respectively. The performance of the proposed system with CR of 4 is the most best.

Comparing Figure 10 with Figure 11, we can see that the sensitivity of the clipped and normalized OFDM system is higher than that of the conventional clipped OFDM system at the difference value of CR. These measured experiment and the former simulation experiment results both verify that the joint data clipping and normalization scheme can greatly improve the BER of systems.

\section{Conclusions}

We proposed a digital clipped and normalized DDO-OFDM system and theoretically described its principles. The BER formula of the proposed scheme is derived for the AWGN channel and fiber channel. Analytical results show that there is an optimum clipping ratio in clipped and normalized

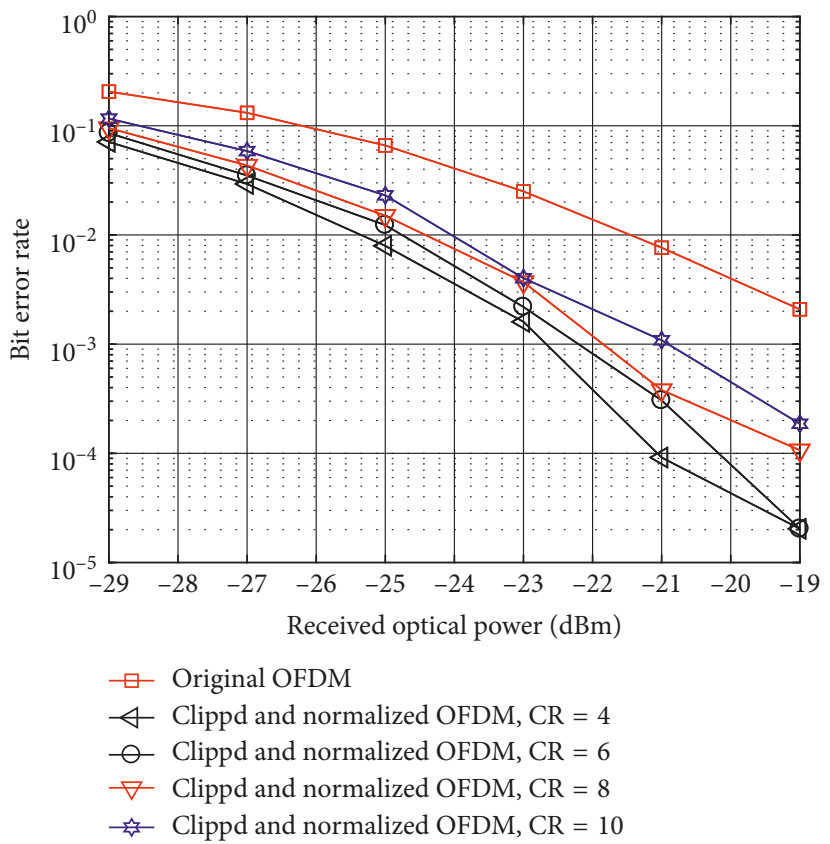

FIGURE 11: Comparison of the BER performance of the clipped and normalized QPSK OFDM signals with the launch power of $6 \mathrm{dBm}$.

OFDM under maximum allowable amplitude constraint. The improvement of a direct detection optical OFDM system achieved by the data clipping and normalization has been assessed experimentally and analyzed theoretically. The measured experimental, simulation, and theoretical analysis results all show that the joint clipping and normalization method can greatly improve the BER of systems. The proposed scheme is an efficient scheme without adding any hardware equipment. For the $4 \mathrm{Gs} / \mathrm{s}$ clipped and normalized QPSK OFDM signal with $\mathrm{CR}=4$, the received sensitivity of the systems can be substantially improved by approximately $4.3 \mathrm{~dB}$ as compared with the conventional OFDM systems. The experimental results also show that the conventional clipped OFDM scheme outperforms the conventional OFDM scheme. Additionally, the main advantage of the proposed scheme is easy implementation in the optical system.

\section{Data Availability}

The data used to support the findings of this study are available from the corresponding author upon request.

\section{Conflicts of Interest}

The authors declare that they have no conflicts of interests.

\section{Acknowledgments}

This work was supported in part by the Zhejiang Provincial Natural Science Foundation of China under LY17F050005, Open Fund of the State Key Laboratory of Millimeter Waves (Southeast University, Ministry of Education, China) under K201214, and Key Laboratory of University Wireless 
Communications (BUPT), Ministry of Education, PR China under KFKT-2018101.

\section{References}

[1] J. Amstrong, "OFDM for optical communication," Journal of Lightwave Technology, vol. 27, no. 3, pp. 189-204, 2009.

[2] J. Armstrong and A. J. Lowery, "Power efficient optical OFDM," Electronics Letters, vol. 42, no. 6, pp. 370-372, 2006.

[3] C. Sánchez, B. Ortega, J. L. Wei, J. Tang, and J. Capmany, "Analytical formulation of directly modulated OOFDM signals transmitted over an IM/DD dispersive link," Optics Express, vol. 21, no. 6, pp. 7651-66, 2013.

[4] W. Shieh, H. Bao, and Y. Tang, "Coherent optical OFDM: theory and design," Optics Express, vol. 16, no. 2, pp. 841-859, 2008.

[5] Y. London and D. Sadot, "Nonlinear effects mitigation in coherent optical OFDM system in presence of high peak power," Journal of Lightwave Technology, vol. 29, no. 21, pp. 3275-3281, 2011.

[6] Y. Gao, J. Yu, J. Xiao, Z. Cao, F. Li, and L. Chen, "Directdetection optical OFDM transmission system with preemphasis technique," Journal of Lightwave Technology, vol. 29, no. 14, pp. 2138-2145, 2011.

[7] S. Azou, Ş. Bejan, P. Morel, and A. Sharaiha, "Performance improvement of a SOA-based coherent optical-OFDM transmission system via nonlinear companding transforms," Optics Communications, vol. 336, no. 4, pp. 177-183, 2015.

[8] C. R. Berger, Y. Benlachtar, R. I. Killey, and P. A. Milder, "Theoretical and experimental evaluation of clipping and quantization noise for optical OFDM," Optics Express, vol. 19, no. 18, pp. 17713-17728, 2011.

[9] M. Sung, J. Lee, and J. Jeong, "DCT-precoding technique in optical fast OFDM for mitigating for fiber nonlinearity," IEEE Photonics Technology Letters, vol. 25, no. 22, pp. 2209-2212, 2013.

[10] L. Cai, Y. Qi, T. Jiang et al., "Investigation of coherent optical multi-band DFT-S OFDM in long haul transmission," IEEE Photonics Technology Letters, vol. 24, no. 19, pp. 1704-1707, 2012.

[11] T. A. Truong, M. Arzel, H. Lin, B. Jahan, and M. Jézéque, "DFT precoded OFDM-an alternative candidate for next generation PONs," Journal of Lightwave Technology, vol. 32, no. 6, pp. 1228-1238, 2014.

[12] J. Xiao, J. Yu, X. Li et al., "Hadamard transform combined with companding transform technique for PAPR reduction in an optical direct-detection OFDM system," Journal of Optical Communications and Networking, vol. 4, no. 10, pp. 709-714, 2012.

[13] H. Chen, J. Yun, J. Xiao, Z. Cao, L. Fan, and L. Chen, "Nonlinear effect mitigation based on PAPR reduction using electronic pre-distortion technique in direct-detection optical OFDM system," Optical Fiber Technology, vol. 19, no. 5, pp. 387-391, 2013.

[14] N. Tu, S. Mhatli, E. Giacoumidis, L. Van Compernolle, M. Wuilpart, and P. Megret, "Fiber nonlinearity equalizer based on support vector classification for coherent optical OFDM," IEEE Photonics Journal, vol. 8, no. 2, pp. 1-9, 2016.

[15] H. Chen and A. M. Haimovich, "Iterative estimation and cancellation of clipping noise for OFDM signals," IEEE Communications Letters, vol. 7, no. 7, pp. 305-307, 2003.

[16] L. Wang and C. Tellambura, "Analysis of clipping noise and tone-reservation algorithms for peak reduction in OFDM systems," IEEE Transactions on Vehicular Technology, vol. 57, no. 3, pp. 1675-1694, 2008.

[17] L. Wang and C. Tellambura, "A simplified clipping and filtering technique for PAR reduction in OFDM systems," IEEE Signal Processing Letters, vol. 12, no. 6, pp. 453-456, 2005.

[18] Z. Yu, R. J. Baxley, and G. T. Zhou, "EVM and achievable data rate analysis of clipped OFDM signals in visible light communication," EURASIP Journal on Wireless Communications and Networking, vol. 2012, no. 1, pp. 1-16, 2012.

[19] L. Nadal, M. S. Moreolo, J. M. Fàbrega, and G. Junyent, "Low complexity PAPR reduction techniques for clipping and quantization noise mitigation in direct-detection O-OFDM systems," Optical Fiber Technology, vol. 20, no. 3, pp. 208-216, 2014.

[20] S. Dimitrov, S. Sinanovic, and H. Haas, "Clipping noise in OFDM-based optical wireless communication systems," IEEE Transactions on Communications, vol. 60, no. 4, pp. 10721081, 2012.

[21] J. Armstrong and B. J. C. Schmidt, "Comparison of asymmetrically clipped optical OFDM and DC-biased optical OFDM in AWGN," IEEE Communications Letters, vol. 12, no. 5, pp. 343-345, 2008.

[22] E. Vanin, "Performance evaluation of intensity modulated optical OFDM system with digital baseband distortion," Optics Express, vol. 19, no. 5, pp. 4280-4293, 2011.

[23] L. Chen, B. Krongold, and J. Evans, "Theoretical characterization of nonlinear clipping effects in IM/DD optical OFDM systems," IEEE Transactions on Communications, vol. 60, no. 8, pp. 2304-2312, 2012.

[24] K. R. Panta and J. Armstrong, "Effect of clipping on the error performance of OFDM in frequency selective fading channels," IEEE Transactions on Wireless Communications, vol. 3, no. 2, pp. 668-671, 2004.

[25] H. Qian, R. Raich, and G. T. Zhou, "On the benefits of deliberately introduced baseband nonlinearities in communication systems," in Proceedings of IEEE International Conference on Acoustics, Speech, and Signal Processing, pp. 905-908, Atlanta, GA, USA, September 2004.

[26] G. Bosco, R. Cigliutti, A. Nespola et al., "Experimental investigation of nonlinear interference accumulation in uncompensated links," IEEE Photonics Technology Letters, vol. 24, no. 14, pp. 1230-1232, 2012.

[27] P. Poggiolini, A. Carena, V. Curri, G. Bosco, and F. Forghieri, "Analytical modeling of nonlinear propagation in uncompensated optical transmission links," IEEE Photonics Technology Letters, vol. 23, no. 11, pp. 742-744, 2011.

[28] A. Demir, "Nonlinear phase noise in optical-fibercommunication systems," Journal of Lightwave Technology, vol. 25, no. 8, pp. 2002-2032, 2007.

[29] H. Chen, J. He, J. Tang et al., "Nonlinear distortion evaluation of MZM with equivalent mathematical model calculation in IM/DD OOFDM transmission system," Optics Communications, vol. 316, pp. 31-36, 2014.

[30] H. Ochiai and H. Imai, "Performance analysis of deliberately clipped OFDM signals," IEEE Transactions on Communication, vol. 50, no. 1, pp. 89-101, 2002.

[31] D. J. F. Barros and J. M. Kahn, "Comparison of orthogonal frequency-division multiplexing an on-off keying in amplified direct-detection single-mode fiber systems," Journal of lightwave technology, vol. 28, no. 12, pp. 1811-1820, 2010.

[32] D. Mestdagh, P. Spruyt, and B. Biran, "Effect of amplitude clipping in DMT-ADSL transceivers," Electronics Letters, vol. 29, no. 15, pp. 1354-1355, 1993. 
[33] J. G. Proakis, Digital Communications, McGraw-Hill, New York, NY, USA, 1995.

[34] A. V. T. Cartaxo and T. M. F. Alves, "Theoretical and experimental performance evaluation methods for DD-OFDM systems with optical amplification," Journal of Microwaves Optoelectronics and Electromagnetic Applications, vol. 10, no. 1, pp. 82-94, 2011.

[35] IEEE Std 802.16a, IEEE Standard for Local and Metropolitan Area Networks-Part 16: Air Interface for Fixed Broadband Wireless Access Systems-Amendment 2: Medium Access Control Modifications and Additional Physical Layer Specifications for 2-11 GHz, IEEE, Piscataway, NJ, USA, 2003.

[36] B. Foo, B. Corcoran, C. Zhu, and A. J. Lowery, "Distributed nonlinearity compensation of dual-polarization signals using optoelectronics," IEEE Photonics Technology Letters, vol. 28, no. 20, pp. 2141-2144, 2016. 


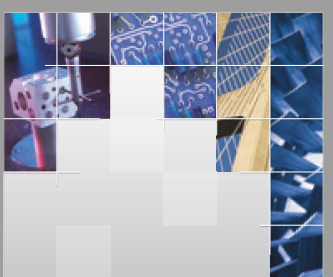

\section{Enfincering}
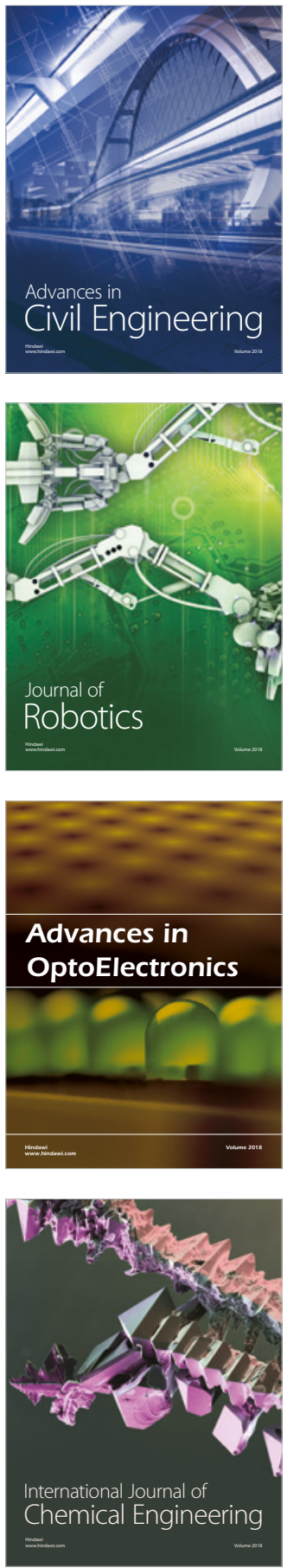

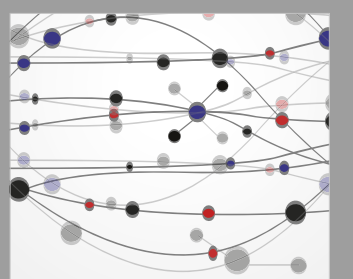

\section{Rotating \\ Machinery}

The Scientific World Journal

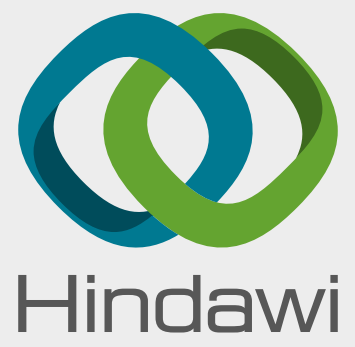

Submit your manuscripts at

www.hindawi.com
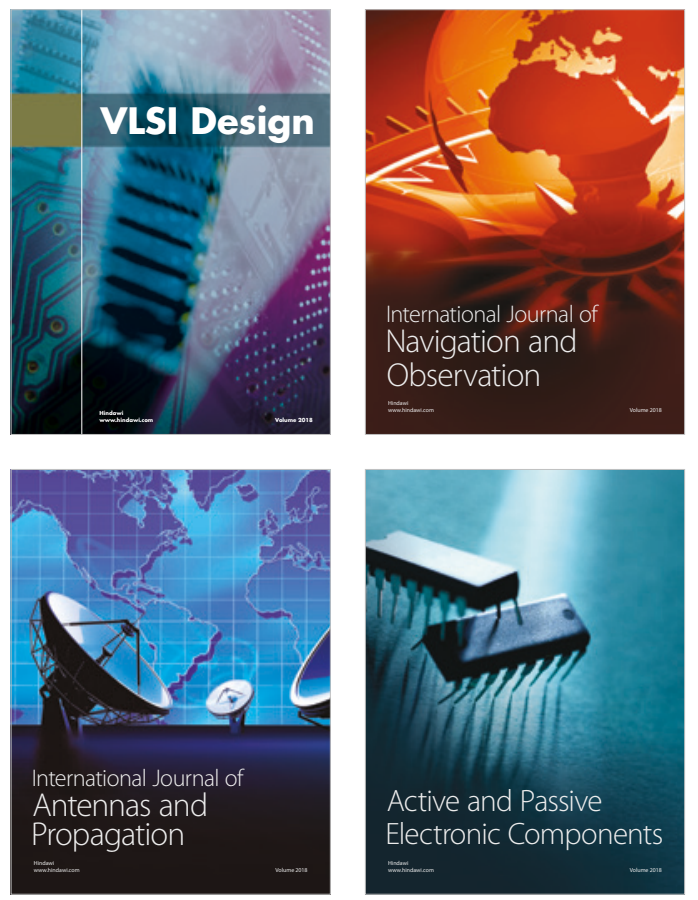
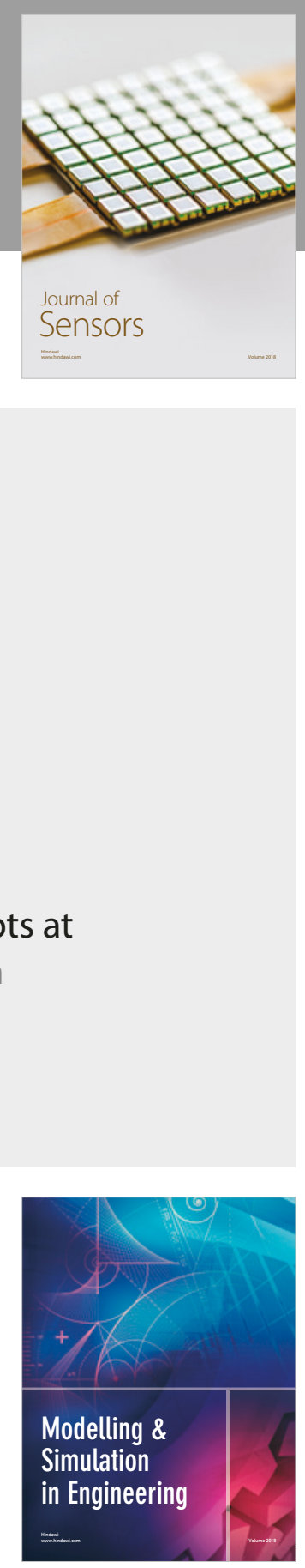

\section{Advances \\ Multimedia}
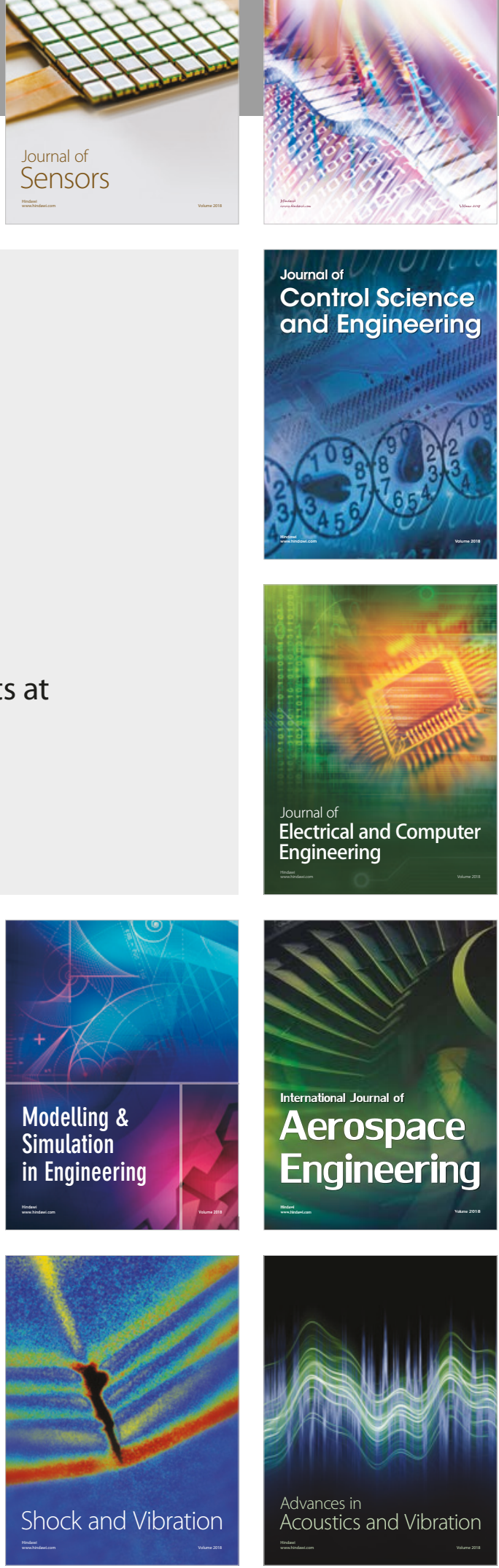